\title{
Spatiotemporal Variability of Turbulence Kinetic Energy Budgets in the Convective Boundary Layer over Both Simple and Complex Terrain
}

\author{
Raj K. Rai, Larry K. Berg, Mikhail Pekour, And William J. Shaw \\ Pacific Northwest National Laboratory, Richland, Washington \\ BRANKO KOSOVIC \\ National Center for Atmospheric Research, ${ }^{\text {a } B o u l d e r, ~ C o l o r a d o ~}$ \\ JEFFREY D. MIROCHA \\ Lawrence Livermore National Laboratory, Livermore, California \\ BRANDON L. ENNIS \\ Sandia National Laboratories, Albuquerque, New Mexico
}

(Manuscript received 6 May 2017, in final form 11 September 2017)

\begin{abstract}
The assumption of subgrid-scale (SGS) horizontal homogeneity within a model grid cell, which forms the basis of SGS turbulence closures used by mesoscale models, becomes increasingly tenuous as grid spacing is reduced to a few kilometers or less, such as in many emerging high-resolution applications. Herein, the turbulence kinetic energy (TKE) budget equation is used to study the spatiotemporal variability in two types of terrain-complex [Columbia Basin Wind Energy Study (CBWES) site, northeastern Oregon] and flat [Scaled Wind Farm Technology (SWiFT) site, west Texas] - using the Weather Research and Forecasting (WRF) Model. In each case, six nested domains [three domains each for mesoscale and large-eddy simulation (LES)] are used to downscale the horizontal grid spacing from $\sim 10 \mathrm{~km}$ to $\sim 10 \mathrm{~m}$ using the WRF Model framework. The model output was used to calculate the values of the TKE budget terms in vertical and horizontal planes as well as the averages of grid cells contained in the four quadrants of the LES domain. The budget terms calculated along the planes and the mean profile of budget terms show larger spatial variability at the CBWES site than at the SWiFT site. The contribution of the horizontal derivative of the shear production term to the total shear production was found to be $\approx 45 \%$ and $\approx 15 \%$ at the CBWES and SWiFT sites, respectively, indicating that the horizontal derivatives applied in the budget equation should not be ignored in mesoscale model parameterizations, especially for cases with complex terrain with $<10-\mathrm{km}$ scale.
\end{abstract}

\section{Introduction}

Accurate representation of the spatial variability of meteorological variables in mesoscale models, such as the Weather Research and Forecasting (WRF) Model (Skamarock et al. 2008) can yield better simulations, particularly over regions with complex terrain. The general formulation of mesoscale models parameterizes

\footnotetext{
${ }^{a}$ The National Center for Atmospheric Research is sponsored by the National Science Foundation.
}

Corresponding author: Raj K. Rai, raj.rai@pnnl.gov turbulence in the vertical direction only; horizontal diffusion is handled separately, such as by using 2D Smagorinsky diffusion. The approach for modeling turbulence assumes statistical homogeneity within the model grid cell. This assumption may be valid for simulations over flat terrain when applying a relatively coarse grid spacing $(>\sim 10 \mathrm{~km})$ that is sufficiently large to allow for an ensemble average over a number of boundary layer eddies. However, when finer horizontal grid spacing is applied in an area of complex terrain, statistical homogeneity is unlikely to occur. As a result, the mesoscale model may not accurately represent the boundary layer turbulence, leading to errors in the 
simulation of mesoscale features of the flow, local weather, and transport and diffusion of air pollutants (air quality studies).

Turbulence kinetic energy (TKE) is widely used to characterize turbulence in space and time. Data from both observations and large-eddy simulations (LES) have been used for this purpose. Wyngaard and Coté (1971) used the TKE budget to study surface layer wind profiles under different stability conditions, and Lenschow (1974) later extended the analysis to altitudes above the surface layer. McBean and Elliott (1975) analyzed the transport terms in the surface layer using the velocity spectra. These and subsequent works have helped to improve the understanding of the production, transport, and destruction of turbulence within the boundary layer. However, they were limited to cases with flat terrain, and statistics have been derived using observations only from a few locations.

Using a highly resolved flow field generated by an LES model, Moeng and Sullivan (1994) studied the vertical distribution of the TKE budget and developed a model of the TKE budget for the shear, buoyancy, and mixed (shear and buoyancy driven) planetary boundary layer (PBL) flows. Over a heterogeneous surface, such as across the forest edge, Yang et al. (2006) studied the TKE budget using LES data and found that the source of TKE is mostly associated with convergence/divergence of mean flow, streamwise advection, pressure transport, and drag. Similarly, a few studies (e.g., Dwyer et al. 1997; Finnigan 2000; Yue et al. 2008) have been conducted using LES to study TKE within the plant canopy. Although these simulations provided data at multiple points for analysis, they were still performed for cases with flat and horizontally homogeneous terrain. For more complex terrain, such as in the case of a simple slope flow (i.e., katabatic), Skyllingstad (2003) studied the TKE budget using LES data. He found that the shear production and dissipation were the dominant terms in the TKE budget and that the buoyancy and pressure transport terms were sufficient to reduce the amount of turbulence above the katabatic jet. Work by Catalano and Moeng (2010) used WRF-LES results to study the characteristics of turbulence in an idealized ridge-valley case and suggested that including the effect of horizontal heterogeneity in the large-scale model could lead to improved forecasts. All of the aforementioned simulations analyzed the TKE budget using a periodic boundary condition in at least one direction, implying that horizontal homogeneity was always present in the direction of the periodicity.

The production and transport of turbulence in horizontal and vertical directions in realistic simulations could be different from those obtained for idealized simulations. With an LES dataset generated using realistic boundary conditions and multiple nested domains, Weigel et al. (2007) studied TKE for a valley case that had grid spacing of $350 \mathrm{~m}$ and compared their results with observations. They found that the shear production term dominated the buoyancy production, and the TKE profile was nicely scaled with $w_{*}$ (Deardorff convective velocity scale) taken from the sunlit side rather than from the base of the valley. However, their work did not analyze the variability of TKE in the entire LES domain, and they also used relatively large grid spacing of $350 \mathrm{~m}$, which may not capture the details of the production, dissipation, and transport of turbulence. Using more highly resolved flow fields can improve our understanding of the spatiotemporal variability in areas of complex as well as simple terrain.

The main objective of this study is to assess spatiotemporal variability in the TKE associated with complex terrain in real-case simulations by analyzing the TKE budget. We use flow fields of meteorological variables generated from multiple nested domains for the convective boundary layer over an area of a complex terrain in northeastern Oregon and over an area of relatively flat terrain in west Texas. For both areas, the outer domains use the WRF model run in mesoscale mode, and inner domains use the WRF Model in LES mode. The results show that the variability of TKE budget terms in the horizontal directions is comparable with that found for the vertical direction, especially for the complex terrain case. A brief discussion of model configuration for both flat and complex terrain simulations is described in section 2. The TKE budget equations and description of budget terms are presented in section 3 . The simulation results are analyzed in the context of the TKE budget in section 4 .

\section{Site selection and model configuration}

Three-dimensional meteorological variables were generated by performing simulations for two sites: the Columbia Basin Wind Energy Study (CBWES) site (Fig. 1a) and the Scaled Wind Farm Technologies (SWiFT) site (Fig. 1b). The surface of the CBWES site is characterized by complex terrain with dryland wheat farms or sage steppe, and the site is located in northeastern Oregon. Simulation results from the CBWES sites have been analyzed in our previous study (Rai et al. 2017). The SWiFT site is located in the southern Great Plains in west Texas and is surrounded by grassland and small bushes. The two distinctly different sitescomplex and flat terrain-were chosen to provide a comparison of the effect of terrain on the terms of TKE budget. Both sites have tall towers equipped with sonic 
a) CBWES site
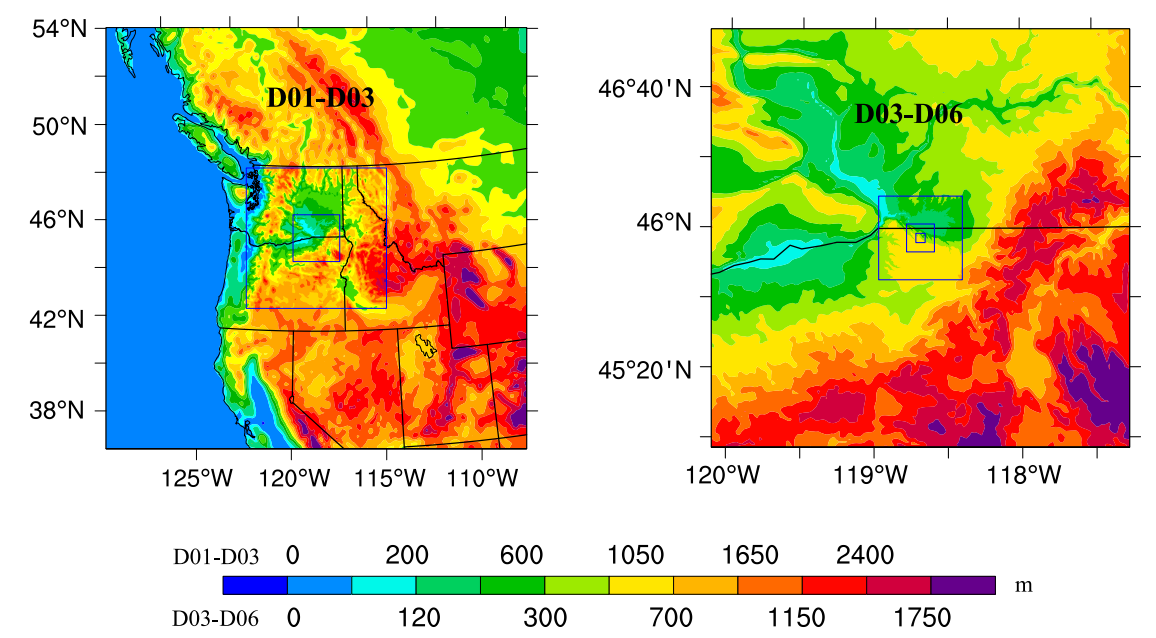

b) SWiFT site
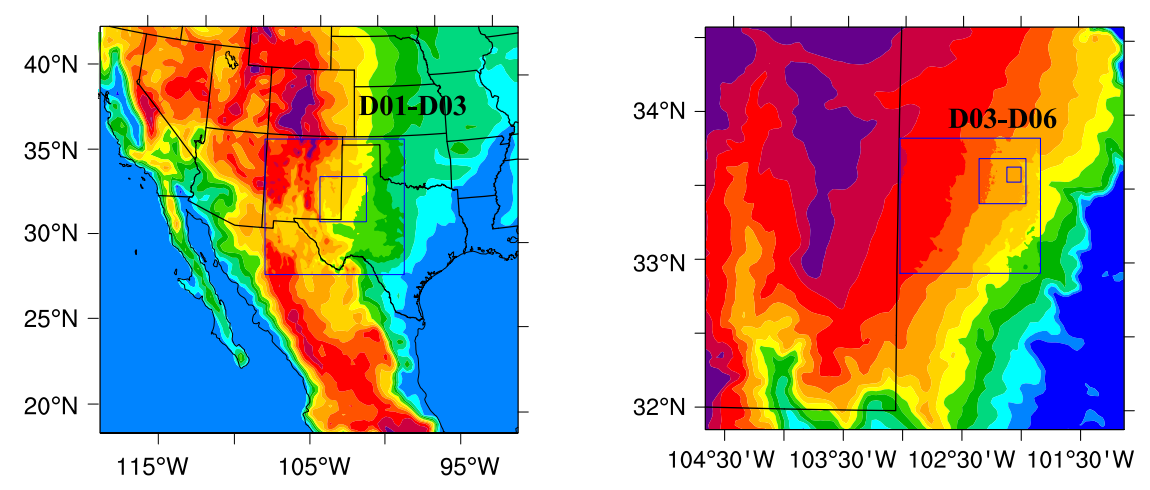

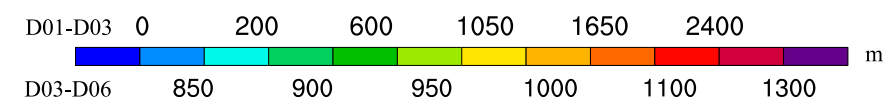

FIG. 1. Configuration for (left) mesoscale domains (D01-D03) and (right) microscale domains (D04-D06) for (a) CBWES and (b) SWiFT with elevation and state boundaries indicated. Note the differences in the color scales between the mesoscale and microscale domains.

anemometers at several heights. The tower at the CBWES site was equipped with three sonic anemometers at two heights (one at $30 \mathrm{~m}$ and the other two at $60 \mathrm{~m}$ ), whereas the Texas Tech University tower at the SWiFT site has 10 sonic anemometers at heights ranging from 0.9 to $200 \mathrm{~m}$. A detailed description for these sites can be found in Berg et al. (2012) and Kelley and Ennis (2016), respectively. Simulations for both sites used six multiple-nested domains (three domains each for mesoscale and microscale) to refine the grid spacing from $\approx 10 \mathrm{~km}$ (in mesoscale) to $\approx 10 \mathrm{~m}$ (in microscale). For the sake of convenience, the simulations in the outermost three domains (D01-D03) and innermost three domains (D04-D06) are henceforth termed as the WRF-Meso model and the WRF-LES model, respectively.

Boundary conditions for the outermost WRF-Meso model domain were provided by the North American Regional Reanalysis (Mesinger et al. 2006). The WRFLES domains were started $15 \mathrm{~h}$ into the WRF-Meso runs (which were started at 1900 PST for the CBWES site and 2100 CST for the SWiFT site) to allow for spinup of the mesoscale model. All WRF-Meso and WRF-LES model domains used the same dynamics and physics parameterizations but had different turbulence parameterizations. Because of its relatively better performance over local schemes, the Mellor-Yamada-Nakanishi-Niino (MYNN) PBL parameterization (Nakanishi and Niino 2006) was 
applied to represent the vertical turbulence stresses and fluxes and their divergence (vertical gradient) in the WRF-Meso model domains. The WRF-LES domains used a three-dimensional turbulence model-with 1.5-order TKE closure (Lilly 1967) — that expressed the eddy viscosity in terms of prognostic subgrid-scale (SGS) TKE. Details of other parameterizations used in the simulations are presented in Table 1. The simulations for the CBWES and SWiFT sites were performed for two days with fair weather-11 May 2011 and 5 May 2014, respectively. The simulations were run concurrently in all six domains using one-way nesting, and the three-dimensional data from the innermost WRF-LES domain were saved every $2 \mathrm{~s}$.

The details of the grid configuration, such as grid spacing and the grid position relative to the parent domain, are different for the two sites. A complete description of the model configuration used for CBWES is presented by Rai et al. (2017), and a similar approach has been used for the simulation of conditions at the SWiFT site. The inner grids at the SWiFT site were shifted northeast (Fig. 1b) to maximize the distance between domain boundaries in the upwind direction at that site. This helps to spin up the turbulence in the northeast region of the inner grid. The simulations over the CBWES site are configured such that all six domains have a common center (Fig. 1a). Details on the grid configuration for both sites are presented in Table 2. The horizontal grid spacing in CBWES site is downscaled from $12 \mathrm{~km}$ (in D01) to $0.03 \mathrm{~km}$ (in D06), and from $30 \mathrm{~km}$ (in D01) to $0.04 \mathrm{~km}$ (in D06) in the SWiFT site. The reasons for choosing different horizontal grid spacing in the simulation of two sites are as follows. The largest PBL depths $z_{i}$, predicted by the WRF Model for the CBWES and SWiFT sites during the afternoon, were found to be approximately 1.2 and $3.8 \mathrm{~km}$, respectively. The horizontal grid spacing for the innermost WRFMeso domain was assigned on the order of $z_{i}$ (i.e., $\Delta x=$ $\Delta y \approx z_{i}$ ) assuming that horizontal grid spacing smaller than approximately $z_{i}$ would fall in the gray zone, as the horizontal grid spacing of approximately $z_{i}$ produces oscillatory values of wind speed in time. Wyngaard (2004) described the gray zone as the range of turbulence scale where eddies are poorly resolved and the grid spacing becomes too small for the standard turbulence parameterization. Furthermore, the horizontal grid spacing for the coarsest WRF-LES domain was also intended to be small enough (i.e., $270 \mathrm{~m}$ in CBWES and $380 \mathrm{~m}$ in SWiFT case). The spatial resolution for the coarsest WRF-LES domains is chosen to avoid the grid spacing falling in the gray zone in both sites. These restrictions of grid spacing resulted in a grid refinement ratio of 5 (for CBWES) and 9 (for SWiFT) between the
TABLE 1. Data and schemes used in WRF-Meso (mesoscale) and WRF-LES (microscale) models.

\begin{tabular}{|c|c|}
\hline PBL schemes & MYNN (Nakanishi and Niino 2006) \\
\hline Radiation schemes & RRTMG (Iacono et al. 2008) \\
\hline Microphysics & $\begin{array}{l}\text { Morrison 2-moment } \\
\quad \text { (Morrison et al. 2009) }\end{array}$ \\
\hline Surface layer scheme & MYNN, MM5 Monin-Obukhov \\
\hline Land surface model & Noah (Chen and Dudhia 2001) \\
\hline $\begin{array}{l}\text { Geographic data } \\
\text { (horizontal resolution) }\end{array}$ & \\
\hline Elevation & $\begin{array}{l}\text { Mesoscale: } 5 \mathrm{~min}, 2 \mathrm{~min}, 30 \mathrm{~s} \\
\text { Microscale: } 1 \mathrm{~s}\end{array}$ \\
\hline Land use & $\begin{array}{l}\text { Microscale and mesoscale: } \\
5 \mathrm{~min}, 2 \mathrm{~min}, 30 \mathrm{~s}\end{array}$ \\
\hline
\end{tabular}

innermost WRF-Meso and the outermost WRF-LES model domains. However, the grid spacing ratio within the WRF-Meso domains and WRF-LES domains was chosen to be 3 in each site. The vertical grid spacing was defined on the basis of the PBL depth, desired grid aspect ratio (ratio of the horizontal to vertical grid spacing), and the finest WRF-LES horizontal resolution. Mirocha et al. (2010) showed the aspect ratio could influence LES results, and they mentioned that a value between 2 and 4 would be ideal for neutral boundary layer flows. Within the PBL, the grid aspect ratio was always restricted to be equal or less than 1 , decreasing from 2 near the surface to almost 1 at the height of the PBL depth. This was done using either a stretched grid or a combination of uniform and stretched grids in the vertical direction (Table 3). For example, for the CBWES site, we use 15-m uniform vertical grid spacing in the first $300 \mathrm{~m}$, and then the grid stretches to the model top; for the SWiFT site, we use two stretched grids: one from $20 \mathrm{~m}$ to $40 \mathrm{~m}$, and the other from $40 \mathrm{~m}$ to the model top.

\section{Analysis approach}

This work uses the TKE budget equation [Eq. (1)], which is derived for an incompressible flow to study the relative contribution of each TKE budget term to the tendency of TKE. The compressibility effect can be ignored, as the boundary layer depth compared to the domain height and the area of the domain considered for the analysis are small:

$$
\begin{aligned}
\frac{\partial\langle e\rangle}{\partial t}+\left\langle\tilde{u}_{j}\right\rangle \frac{\partial\langle e\rangle}{\partial x_{j}}= & \delta_{i, 3} \frac{g}{\left\langle\tilde{\theta}_{v}\right\rangle}\left\langle\tilde{u}_{i}^{\prime} \tilde{\theta}_{v}^{\prime}+u_{i}^{\prime} \theta_{v}^{\prime}\right\rangle-\left\langle\tilde{u}_{i}^{\prime} \tilde{u}_{j}^{\prime}+u_{i}^{\prime} u_{j}^{\prime}\right\rangle \\
& \times \frac{\partial\left\langle\tilde{u}_{i}\right\rangle}{\partial x_{j}}-\frac{\partial}{\partial x_{j}}\left(\left\langle\tilde{u}_{j}^{\prime} e\right\rangle+\frac{1}{\rho_{0}}\left\langle\tilde{u}_{j}^{\prime} \tilde{p}\right\rangle\right)+\varepsilon
\end{aligned}
$$

The first and second terms on the left-hand side of the TKE equation represent the tendency of TKE (or local) 
TABLE 2. Domain size, grid spacing, and time step size used in WRF-Meso-LES simulation for SWiFT and CBWES sites.

\begin{tabular}{|c|c|c|c|c|c|c|c|}
\hline Terrain & Center & Domain (D0x) & Model & $\Delta x, \Delta y(\mathrm{~km})$ & $\mathrm{nx} \times \mathrm{ny}$ & $\mathrm{nz}$ & $\Delta t(\mathrm{~s})$ \\
\hline \multirow[t]{6}{*}{ Complex } & $45.9550^{\circ} \mathrm{N},-118.6876^{\circ} \mathrm{W}$ & 1 & Mesoscale & 12.15 & $166 \times 166$ & 140 & 15 \\
\hline & & 2 & & 4.05 & & & 5 \\
\hline & & 3 & & 1.35 & & & $5 / 3$ \\
\hline & & 4 & LES & 0.27 & & & $1 / 3$ \\
\hline & & 5 & & 0.09 & & & $1 / 9$ \\
\hline & & 6 & & 0.03 & & & $1 / 27$ \\
\hline \multirow[t]{6}{*}{ Flat } & $33.6105^{\circ} \mathrm{N},-105.0505^{\circ} \mathrm{W}$ & 1 & Mesoscale & 30.62 & $100 \times 91$ & 154 & 18 \\
\hline & & 2 & & 10.21 & & & 6 \\
\hline & & 3 & & 3.40 & & & 2 \\
\hline & & 4 & LES & 0.38 & $298 \times 271$ & & $1 / 3$ \\
\hline & & 5 & & 0.13 & $298 \times 271$ & & $1 / 9$ \\
\hline & & 6 & & 0.04 & $271 \times 271$ & & $1 / 27$ \\
\hline
\end{tabular}

term and advection term, respectively; the terms on the right-hand side, in order, are buoyancy production, shear production, transport (turbulent and pressure), and dissipation (sink) terms. Usually, in unstable conditions, the first two terms (i.e., buoyancy and shear production) represent the generation of turbulence. Transport terms do not generate turbulence but move the turbulence from one location to another location. Within the LES, the dissipation term $\varepsilon\left(=2\left\langle u_{i}^{\prime} u_{j}^{\prime} \tilde{S}_{i j}\right\rangle\right)$ is estimated as the energy transferred from the resolved scale to the subgrid scale because the viscous dissipation for a high Reynolds number flow in the resolved part of the inertial subrange is negligible. In Eq. (1), the scalar variable $\phi=\left[u_{i}\right.$ (velocity), $\theta_{v}$ (virtual potential temperature), $p$ (pressure)] can be expressed as $\phi=\tilde{\phi}+\phi^{\prime}$, where $\tilde{\phi}$ is the resolved scalar variable obtained after applying a filtering function $G(r)$ (herein, box filter) in the flow field as $\tilde{\phi}=\int G(r) \phi d r$, and $\phi^{\prime}$ is the fluctuation about $\tilde{\phi}$ with the property $\left\langle\phi^{\prime}\right\rangle \neq 0$. The anglebracket operator represents temporal averaging to any scalar variable $\phi$. The instantaneous TKE $e$ is expressed as $0.5\left(\tilde{u}_{i}^{\prime} \tilde{u}_{i}^{\prime}+u_{i}^{\prime} u_{i}^{\prime}\right)$, where $\tilde{u}_{i}^{\prime}=\tilde{u}_{i}-\left\langle\tilde{u}_{i}\right\rangle$. The symbols $\rho_{0}$ and $\tilde{S}_{i j}$ denote the constant density and strain-rate tensor of the resolved velocity, respectively. The products of fluctuating quantities $u_{i}^{\prime} u_{j}^{\prime}$ and $u_{i}^{\prime} \theta_{v}^{\prime}$ are the SGS stress tensor and heat flux, respectively. The variables $t$ and $x_{j}(j=1-3)$ are the independent variables for the time and the three Cartesian spatial directions.

Three-dimensional meteorological variables from WRF-LES domain D06 were used to analyze each term of Eq. (1). Figures $2 a$ and $2 b$ show the contour of terrain elevation over an area of WRF-LES domain D06 for CBWES and SWiFT sites, respectively. It shows that the change in terrain elevation in the complex terrain site is approximately $300 \mathrm{~m}$, whereas it is approximately $10 \mathrm{~m}$ in the flat terrain site. A subset of points, (i.e., a-e from the SE and SW quadrants for CBWES and SWiFT sites) and transects (i.e., $l-l$ and $r-r$ ) are highlighted and will be used to examine results in subsequent sections. Transects $l-l$ and $r-r$ were chosen purposely such that the mean wind direction in each site aligns along and across two transects. Note that transect $r-r$ also aligns to the ridge in the CBWES site. In addition to transects, the horizontal and vertical lines divide the WRF-LES region of both the CBWES (Fig. 2a) and SWiFT (Fig. 2b) sites into quadrants [i.e., southwest (SW), northwest (NW), northeast (NE), and southeast (SE)]; each represents a typical convection-resolving resolution $(2.5 \mathrm{~km} \times 2.5 \mathrm{~km}$ in CBWES and $3.4 \mathrm{~km} \times 3.4 \mathrm{~km}$ in SWiFT $)$. Similarly, data from the sixth model layer (i.e., $x-y$ plane view at $\approx 72 \mathrm{~m}$ above the surface) of the CBWES site are also used for the analysis. This height or model layer was chosen from the perspective of wind energy applications as it is near the typical hub height. The temporal averaging interval of $30 \mathrm{~min}$ was used to calculate the TKE budget terms. The spatial derivative of the scalar quantity $\partial \phi / \partial x_{i}$ in Eq. (1) was computed using a centered finite difference scheme.

\section{Results and discussion}

Before presenting results of the analysis of TKE budget terms, comparisons are made between the mean velocity, TKE, and velocity spectra derived from simulations and

TABLE 3. Details of vertical grid spacing for SWiFT and CBWES sites. The $z_{i, \max }$ is the maximum boundary layer depth for the analysis time period.

\begin{tabular}{lcccrc}
\hline \hline Terrain & Domain $(\mathrm{D} 0 x)$ & $\Delta z(\mathrm{~m})$ & $z$ range $(\mathrm{km})$ & $\mathrm{nz}$ & $z_{i, \max }(\mathrm{km})$ \\
\hline Complex & $1-6$ & 15 & $0-0.3$ & 20 & 1.2 \\
& & $15-30$ & $0.3-2$ & 72 & \\
\multirow{2}{*}{ Flat } & & $30-950$ & $2-$ top & 49 & \\
& $1-6$ & $20-40$ & $0-4$ & 105 & 3.8 \\
& & $40-950$ & $4-$ top & 50 & \\
\hline
\end{tabular}



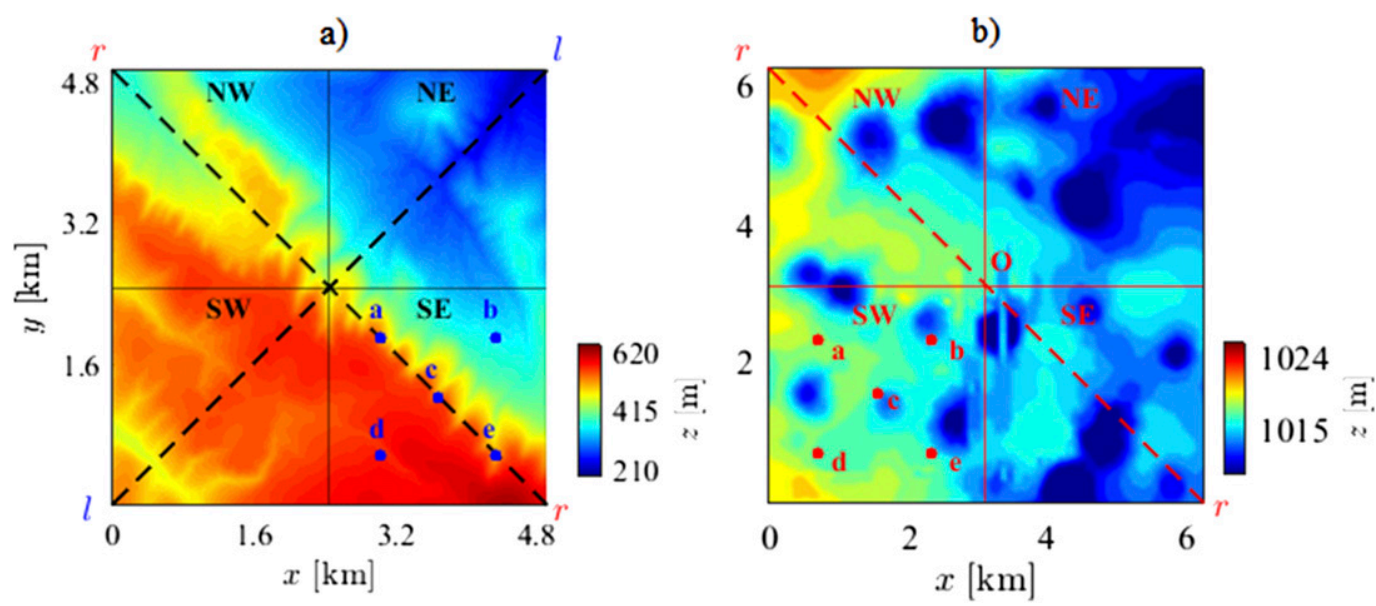

FIG. 2. Contours of terrain elevation of LES domain D06 for (a) complex terrain (CBWES) and (b) flat terrain (SWiFT). The dashed lines $l-l$ and $r-r$ and points a-e show the locations for transects of vertical planes and vertical profiles of TKE budget terms, respectively.

observations. These comparisons are made to demonstrate how well the WRF Model predicted the meteorological variables compared to the observations. Analysis of budget terms is performed in a number of vertical profiles and planes to understand the spatial variability of the terms.

\section{a. Wind characterization}

To evaluate the variables predicted by the two simulation sites (CBWES and SWiFT), the first- and secondorder of statistical moments [i.e., wind speed $\left(\tilde{u}_{1}^{2}+\tilde{u}_{2}^{2}\right)^{1 / 2}$, TKE $\left.\left(\tilde{u}_{i}^{\prime} \tilde{u}_{i}^{\prime} / 2\right)\right]$ and spectra of the $u$ and $w$ components of wind velocity (spectra of $v$ component of wind velocity not shown here) derived from simulations are compared with observations. Figures $3 \mathrm{a}$ and $3 \mathrm{~b}$ and Figs. $3 \mathrm{~d}$ and $3 \mathrm{e}$ show the simulated and observed (sonic) time series of the mean wind speed and TKE for the CBWES site $(z=60 \mathrm{~m})$ and SWiFT site $(z=72 \mathrm{~m})$, respectively, at the location of the tower in domain D06. The time series for the TKE and mean wind speed were derived from averages over $10 \mathrm{~min}$ of instantaneous model output. The results show that the simulated TKE and wind speed are generally found to be in good agreement with observations, with a maximum discrepancy of $\approx 2 \mathrm{~m} \mathrm{~s}^{-1}$. The TKE values are found to be larger at the SWiFT site, which is likely a function of conditions on that particular day. In particular, the surface peak heat flux predicted by the WRF Model at the SWiFT site is $\sim 400 \mathrm{~W} \mathrm{~m}^{-2}$, whereas the surface heat flux at the CBWES site is $\sim 150 \mathrm{~W} \mathrm{~m}^{-2}$. Similarly, the wind speed slightly increases around 1200 CST at the SWiFT site, whereas it decreases continuously throughout the day for the CBWES site. Furthermore, the spectra for the $u$ and $w$ components of wind velocity calculated for both sites (Figs. 3c,f) demonstrate that the WRF Model resolves turbulence up to its effective resolution $\approx 7 \Delta x$ (Skamarock 2004). The spectra were computed by averaging four individual spectra that were constructed from each 30-min time series (i.e., 0-30, 30-60, 60-120, and $120-180 \mathrm{~min}$ ). For both simulated and observed data, the sampling frequency used was $0.5 \mathrm{~Hz}$. The result shows that the velocity spectra simulated by the model are in good agreement with the velocity spectra derived from observations at each site. The spectra for the $v$ component also exhibit similar results to the $u$-component spectra (not shown). The spectra for the $w$ component show less power in both sites than those of the $u$ component, as expected. However, the power in the spectra from the SWiFT site is found to be larger than that from the CBWES, suggesting higher TKE in the SWiFT site. A comprehensive analysis (statistical moments, spectra, probability density function, etc.) of WRF-LES data for CBWES site has been performed in previous work (Rai et al. 2017) and is not investigated further here. Overall, the first- and second-order statistical moments, as well as the power spectra computed at the center of WRF-LES domain D06 for both sites, are well predicted by the WRF Model.

\section{b. Gradients of turbulent stresses}

In mesoscale models, the derivative of the variances and covariances (i.e., tendencies) are parameterized differently in the vertical and horizontal directions. For example, the vertical derivative of the covariances is used in the MYNN PBL parameterization (Nakanishi and Niino 2006), whereas the horizontal deformation is used as a part of the horizontal diffusion (Smagorinsky 1963) in WRF-Meso. The different schemes (i.e., PBL parameterization and diffusion) used in parameterizing the turbulence are based in part on the assumption of 

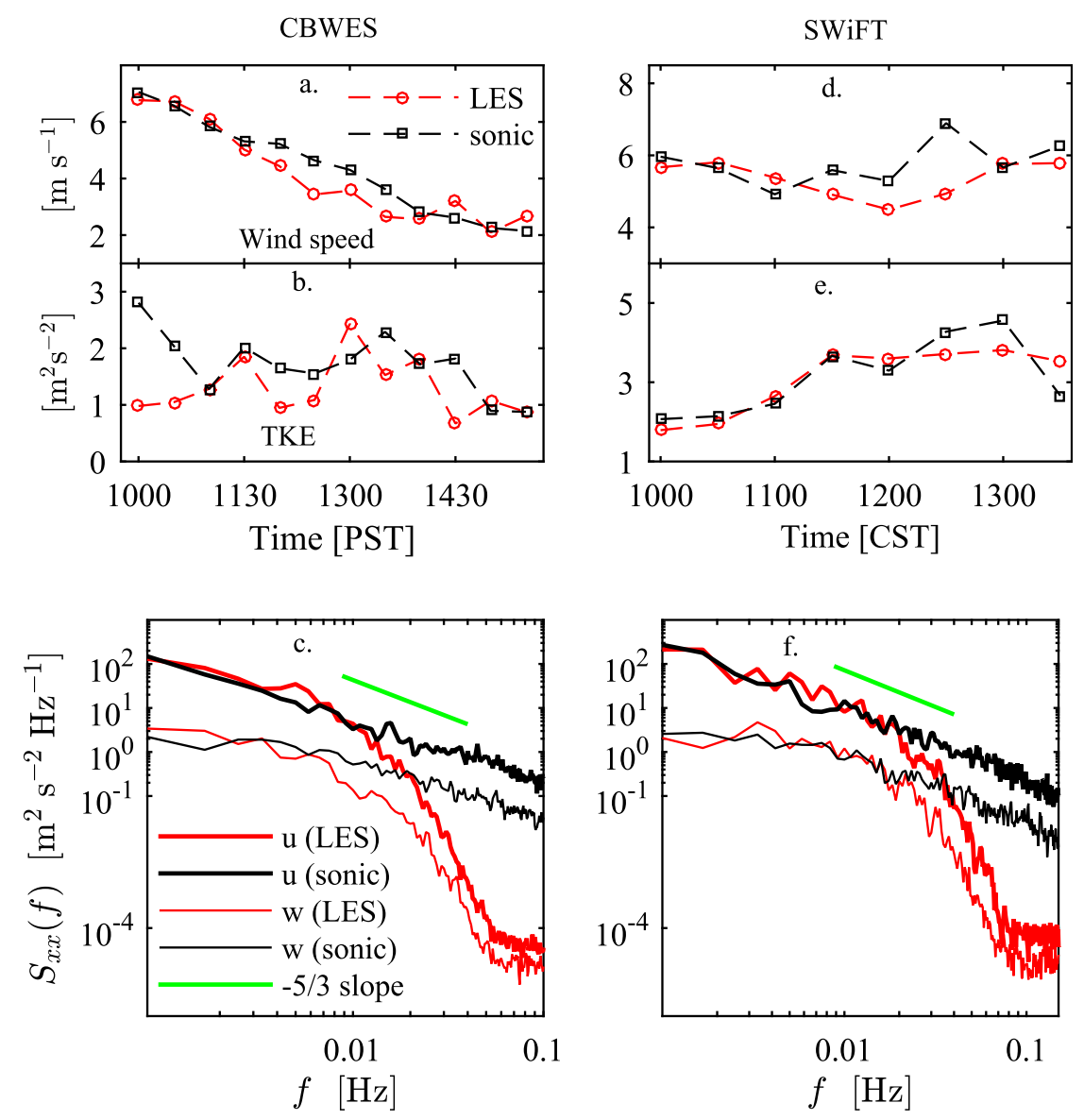

FIG. 3. Comparison of mean wind speed, TKE, and spectra of $u$-and $w$-velocity components for (a)-(c) CBWES and (d)-(f) SWiFT between the simulation data and observations. Note that the velocity spectra for the $w$ component has been reduced by an order of magnitude relative to that for the $u$ component.

ensemble averaging and statistical homogeneity in the horizontal direction. To examine the variation of turbulence stresses $\left(\left\langle\tilde{u}_{i}^{\prime} \tilde{u}_{j}^{\prime}\right\rangle\right.$ for $\left.i, j=1-3\right)$ along the vertical and horizontal directions, the spatial derivatives of turbulence fluxes were computed using 30-min data of the resolved wind field and SGS stresses from the WRF-LES model. Figure 4 shows the spatial derivative of stresses $\left\langle\tilde{u}_{i}^{\prime} \tilde{u}_{j}^{\prime}\right\rangle / \partial x_{k}$, where $k=1-3$ for the CBWES site (left column) and SWiFT site (right column), respectively, at five locations (a-e, see Fig. 2); these locations are $90 \mathrm{~m}$ above the surface in each quadrant for the innermost WRF-LES model domain D06. The derivative of stresses was normalized by $w_{*}^{2} / z_{i}$, where the scaling parameters are the Deardorff convective velocity

$$
w_{*}=\left[g z_{i}\left\langle\tilde{u}_{3}^{\prime} \tilde{\theta}_{v}^{\prime}+u_{3}^{\prime} \theta^{\prime}\right\rangle /\left\langle\theta_{v}\right\rangle\right]^{1 / 3}
$$

and boundary layer depth $z_{i}$. The symbols $g$ and $\theta_{v}$ denote gravitational acceleration and virtual potential temperature. The value of $z_{i}$ was obtained from the standard WRF-Meso output. A centered difference scheme was applied to calculate the derivatives of the turbulence fluxes. As expected, the result shows that the vertical derivative of the various stresses (i.e., $\left\langle\tilde{u}_{i}^{\prime} \tilde{u}_{j}^{\prime}+u_{i}^{\prime} u_{j}^{\prime}\right\rangle / \partial x_{k}$ for $i=1-3, j=3$, and $k=3$ ) has a larger magnitude at both sites, compared to the horizontal derivatives. Among these three vertical derivatives (i.e., $\left\langle\tilde{u}_{1}^{\prime} \tilde{u}_{3}^{\prime}+u_{1}^{\prime} u_{3}^{\prime}\right\rangle / \partial x_{3},\left\langle\tilde{u}_{2}^{\prime} \tilde{u}_{3}^{\prime}+u_{2}^{\prime} u_{3}^{\prime}\right\rangle / \partial x_{3}$, and $\left.\left\langle\tilde{u}_{3}^{\prime} \tilde{u}_{3}^{\prime}+u_{3}^{\prime} u_{3}^{\prime}\right\rangle / \partial x_{3}\right)$, the third one is neglected in the MYNN PBL parameterization. However, the results show that the magnitude of $\left\langle\tilde{u}_{3}^{\prime} \tilde{u}_{3}^{\prime}+u_{3}^{\prime} u_{3}^{\prime}\right\rangle / \partial x_{3}$ is comparable to the first two derivatives. With the exception of $k=2$ for the SWiFT site, all other horizontal derivatives exhibit smaller magnitudes in all locations and quadrants compared to the vertical derivatives $(k=3)$. Nevertheless, the magnitudes for all the horizontal derivatives ( $k=1$ and 2$)$ are important and show considerable spatial variability of the turbulence stresses 


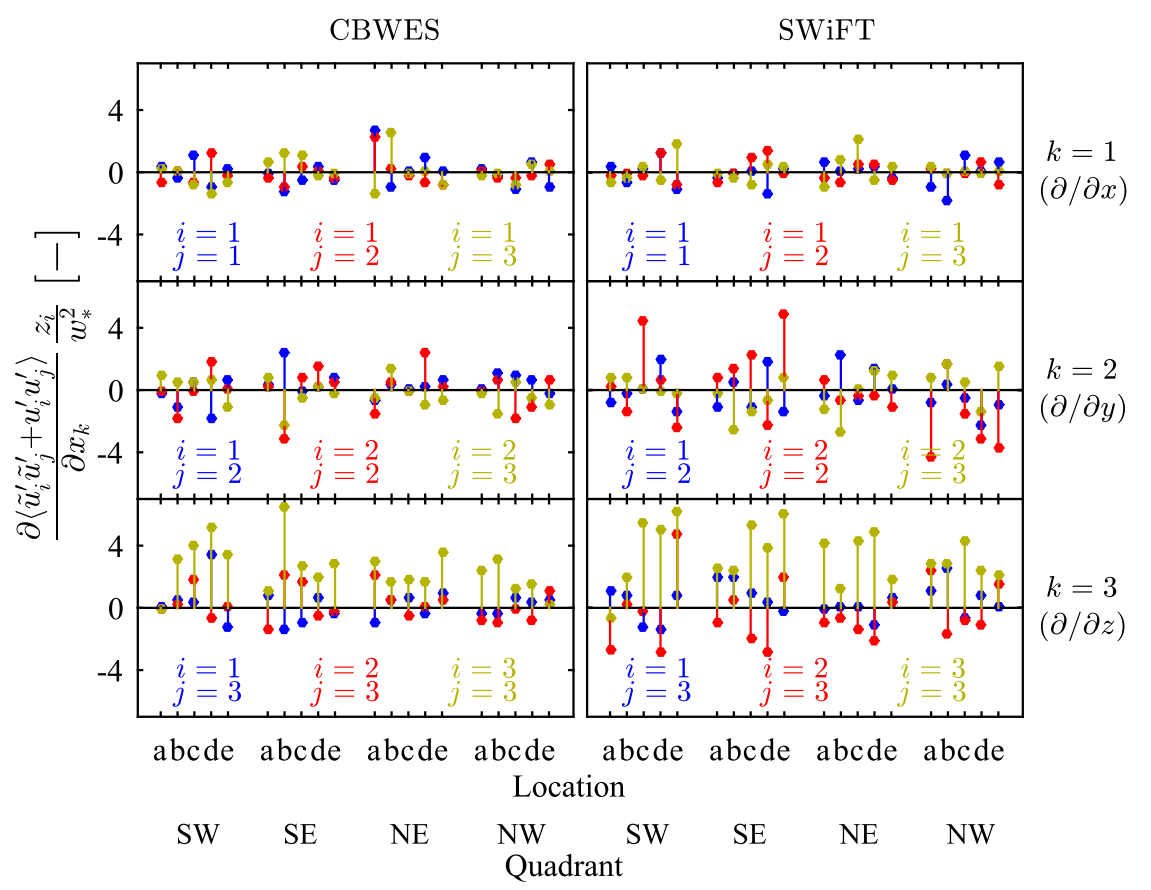

FIG. 4. Gradients of variance and covariance of velocity components at five locations (a-e) in quadrants SW, SE, NE, and NW for (left) CBWES and (right) SWiFT. Values for $i$ and $j$ $(=1-3)$ denote the velocity components, whereas $k(=1-3)$ signifies the derivative with respect to space.

across the WRF-LES domain. The spatial variability seen in the horizontal direction implies the necessity of parameterizing turbulence in all directions. Moreover, comparisons of the results from both sites do not display any noticeable differences that could be attributed to the topography. Note that the elevation differences across the CBWES and SWiFT sites are approximately 400 and $10 \mathrm{~m}$, respectively. Similarly, the magnitude of horizontal derivative of the stresses for both sites shows that the PBL turbulence is horizontally inhomogeneous. The horizontal homogeneity that results from an idealized LES (which uses periodic lateral boundary) over a flat homogeneous surface may not be representative of the SWiFT site, although this site is relatively flat and characterized by similar vegetation within the LES domain. Our simulations of the SWiFT site are not idealized, mainly because of the nonperiodic boundary conditions and the (albeit relatively small) variations in the terrain height.

\section{c. TKE budget profiles}

The spatial variability seen in the turbulent fluxes and their spatial derivatives for a daytime convective case is associated with the amount of turbulence that is produced, transported, and dissipated in space and time. The relative contribution of these processes to the net turbulence can be assessed through an analysis of the TKE budget terms [Eq. (1)]. The left and right panels of
Fig. 5 present the vertical profiles of the TKE and various budget terms, except for the term for pressure transport, at five locations (i.e., a-e) in the SE quadrant for the CBWES site and three locations (i.e., a-c) in the SW quadrant for the SWiFT site, respectively. The vertical profiles were derived from $30 \mathrm{~min}$ of data generated from WRF-LES for domain D06 for the period 1300-1330 PST (at the CBWES site) and 1200-1230 CST (at the SWiFT site). We did not analyze the pressure transport term because the divergence of the simulated wind field $\left(\partial\left\langle\tilde{u}_{i}^{\prime}\right\rangle / \partial x_{i}\right)$ was found to be on the order of $10^{-2} \mathrm{~s}^{-1}$, suggesting that the pressure is tightly coupled with the density. Use of the decoupled pressure for deriving the pressure transport term (formulated for incompressible flow) would likely result in erroneous results. To evaluate the relative contribution of each term associated with the horizontal and vertical derivatives to the sum (horizontal plus vertical derivative), the relative contribution from the horizontal derivative (Hor; $\partial\langle\rangle / \partial x+\partial\langle\rangle / \partial y$ ), the vertical derivative (Ver; $\partial\langle\rangle / \partial z)$, and the sum of horizontal and vertical derivatives (Tot) of each budget term is calculated and then normalized using

$$
w_{*}^{3} / z_{i}=\left[g\left(\left\langle\tilde{u}_{3}^{\prime} \tilde{\theta}_{v}^{\prime}+u_{3}^{\prime} \theta^{\prime}\right\rangle\right)_{s} /\left\langle\tilde{\theta}_{v}\right\rangle\right] .
$$

The vertical distance was also normalized by $z_{i}$. 


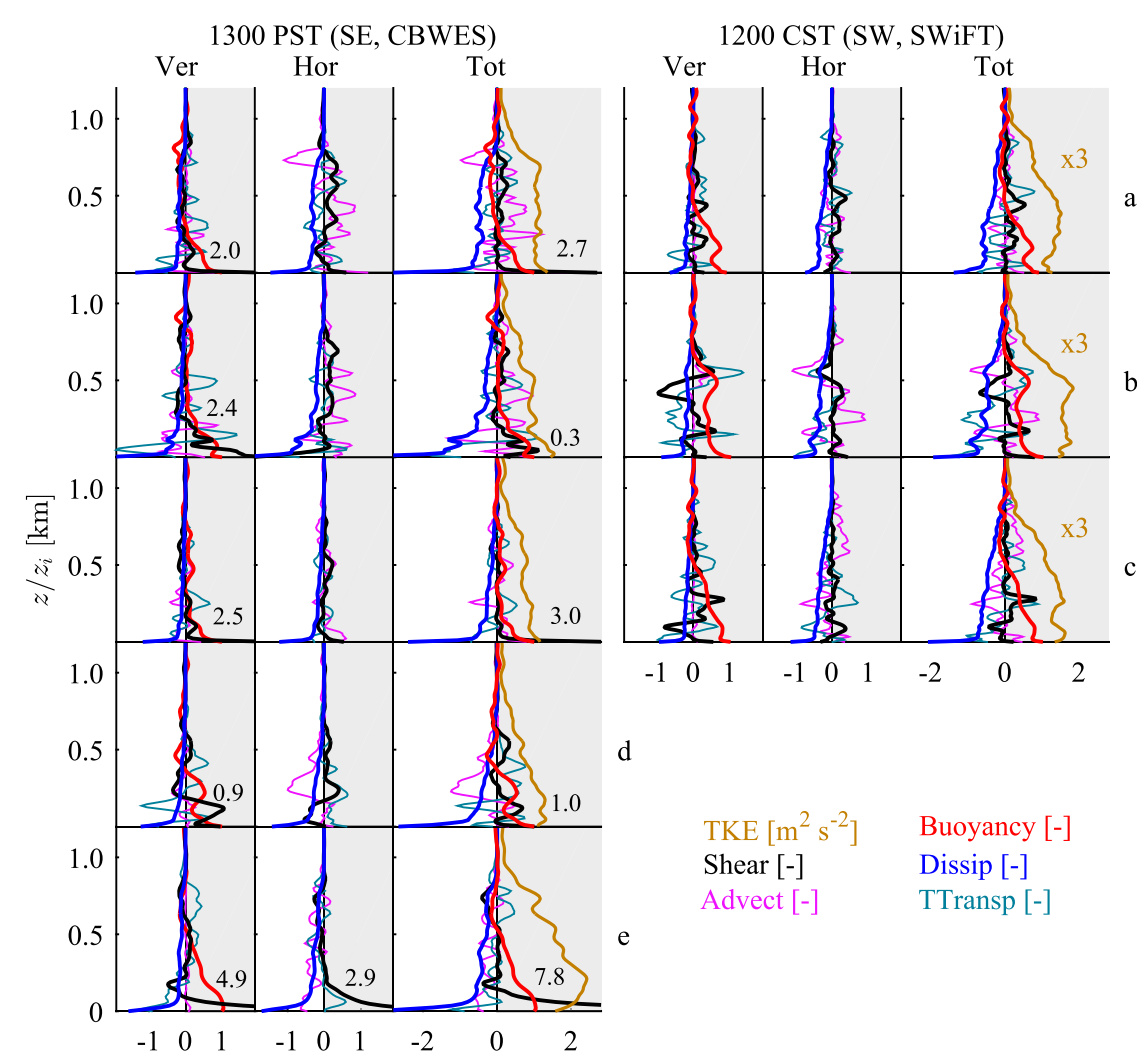

FIG. 5. Vertical profile of TKE and budget terms derived from LES data at (left) five locations (a-e) for the period of 1300-1330 PST (CBWES, SE quadrant) and (right) three locations (a-c) for period of 1200-1230 CST (SWiFT, SW quadrant). The labels Ver, Hor, and Tot represent the terms obtained using vertical, horizontal, and their sum. Gray and white areas in the plot show the positive and negative value of the budget term, respectively. The number in black shows the shear term at the first grid near the surface, and the number in orange $(\times 3)$ denotes that the TKE should be multiplied by 3 .

Previous TKE budget studies (e.g., Moeng and Sullivan 1994) neglected the horizontal terms because they imposed periodic boundary forcing in the horizontal direction, resulting in horizontal homogeneity. Based on our analysis, the profiles of the budget terms (Fig. 5, left and right panels) in both sites show that the horizontal and vertical derivatives associated with the budget terms are comparable in magnitude. Similarly, in most of the locations within the domain, the production terms-shear and buoyancy-computed near the surface have the largest magnitude, as documented by others (e.g., Stull 1988). However, a detailed examination of the profiles of the budget terms in the CBWES and SWiFT sites reveals important differences. For example, shear production is the dominant budget term close to the surface (Fig. 5, left panel) at all five locations within the CBWES domain. Furthermore, the shape and magnitude of the TKE profiles at the different locations (a-e) within the CBWES site are also found to be different-with location e showing the largest TKE, caused by both large amounts of shear and buoyancy production. Additionally, the influence of terrain in the CBWES domain is evident in the total shear production (e.g., Tot) of locations a (shear 2.7), c (shear 3.0), and e (shear 7.8), as these locations lie along the ridge ( $r-r$ transect) that runs through the domain. When considering only the production terms (i.e., shear and buoyancy), both production terms are dominant at location $\mathrm{d}$ (far from the ridge), whereas only the buoyancy production term is dominant at location a (near the ridge). Catalano and Moeng (2010) also observed differences in the vertical profile of TKE budget terms in various locations for their idealized valley case. In the SWiFT domain (Fig. 5, right panel), the buoyancy production term is found to be dominant throughout the depth of the PBL. Similarly, the shape and magnitude of the TKE profiles is comparable in all locations (e.g., rows a-c in the right panel of Fig 5; rows d and e are not shown), within that study domain. The comparison of budget profiles between complex and flat terrains shows a great 

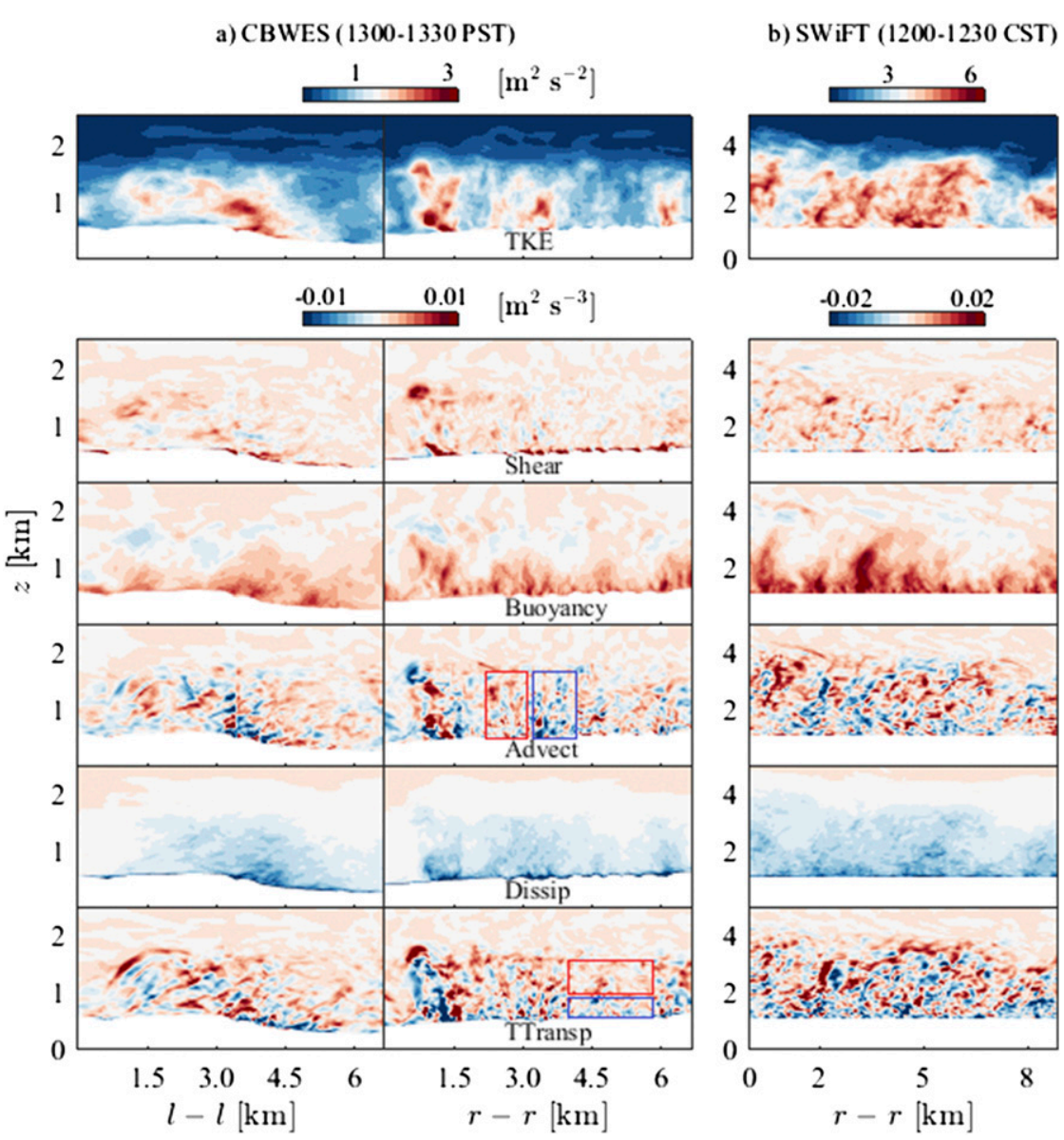

FIG. 6. Contours of TKE and budget terms-shear (Shear), buoyancy (Buoyancy), advection (Advect), dissipation (Dissip), and turbulent transport (TTransp) - in the vertical planes passing through transects $l-l$ and $r-r$ for (left) CBWES and (right) SWiFT. The red and blue boxes are used to show the intensity of the TKE budget terms.

deal of spatial variability in the budget terms, such as the large shear production term near the ridge in the CBWES site.

The budget terms calculated in vertical planes (i.e., contour of budget terms) reveal additional details, such as turbulence scale and intensity of turbulence variability within the boundary layer. Figure 6 a presents the contour of TKE (first row) and budget terms (second through sixth rows) for the CBWES site (1300-1330 PST) in the vertical plane along transects $l-l$ (along the mean wind direction) and $r-r$ (across the mean wind direction). The results show significant TKE variability in the vertical plane along the two transects. The shear production term in the $l-l$ transect has large positive values close to the surface where the terrain slope is maximum. Similarly, the buoyancy production in the vertical plane along the $l-l$ transect shows larger structures (plumes) that are mostly confined to an area near the largest terrain slope and locations where small amounts of subsidence is seen within the boundary layer (negative mean vertical velocity, not shown here). For a steep valley case, Weigel et al. (2007) found that the shear production provided the largest contribution to the TKE, followed by the buoyancy production in the afternoon. In their study, the contours in vertical planes for the production terms (shear and buoyancy) showed a large spatial variability, mostly located near the base of the valley. In the present work, the advection and turbulence transport are more active throughout the boundary layer, and they tend to be large where the production terms (i.e., shear and buoyancy) are large. The pattern of positive (red) and negative (blue) values in the contour plot of advection and turbulent transport reveals that the movement of turbulence occurs in both horizontal and vertical directions, but their contribution is fairly noisy. As expected, the dissipation term is the largest in the region where the production terms (i.e., shear and buoyancy) are also large. The magnitude of 
TKE close to the surface is generally small, except in the region of sloped terrain at a distance of $\approx 4.5 \mathrm{~km}$ into the domain. Less turbulence near the surface may have resulted from turbulence, either because of it being transported to another location (such as vertical transport from lower- to upper-boundary layer) or it being dissipated locally.

The budget terms in the plane along the transect $r-r$ (Fig. 6a, right column) show similar behavior to that observed along the transect $l-l$. However, the orientation of structures along this transect is different for three reasons: 1) the plane is perpendicular to the mean wind direction, 2) the vertical plane is nearly equidistant from the inflow boundaries, and 3 ) the surface elevation in this plane is relatively uniform because it is the top of the ridge. The structures in each budget term in transect $r-r$ are oriented vertically rather than stretched horizontally, as seen in transect $l-l$. The shear production term adjacent to the surface $(\approx 3-\mathrm{km}$ distance from left $)$ in transect $r-r$ shows large positive values, which provide evidence of large spatial variability of turbulence (variability on the length scale of $\sim 100 \mathrm{~m}$ or so). The vertical plane of the TKE advection shows that the advection of turbulence is occurring mostly in the horizontal direction, such as it is occurring in the horizontal direction across the vertical blue and red boxes in Fig. 6a. On the other hand, the turbulent transport in transect $r-r$ is largely active in the vertical direction, such as shown in the horizontal blue and red boxes in Fig. 6a. In contrast, the plane along transect $l-l$ shows the advection and turbulence transport are more active in both directions than in transect $r-r$, which may be due to the sloped terrain and the flow along mean wind through the transect.

Compared to the CBWES results, the spatial distribution of TKE and budget terms for the SWiFT site are expected to be more uniform because of the simpler terrain. Figure $6 \mathrm{~b}$ presents the TKE and budget terms in a vertical plane along transect $r-r$ (across the mean wind direction) calculated using data for the time period 1200-1230 CST. The results show the structures in the TKE and budget terms for the SWiFT site are more homogeneous in space compared to CBWES site. For example, there are no prominent structures close to the surface in the shear production term (although the wind speed and surface roughness is larger than the CBWES site). The dissipation term shows less variation along the plane, which is similar to the TKE contour exhibited in the top of Fig. 6 b. This indicates the homogeneity of structures within the boundary layer in the SWiFT site. Although not shown here for brevity, the vertical contour plane through the transect perpendicular to the transect $r-r$ shows structures that are stretched along the mean wind direction, but there is not as much spatial variability as observed in the CBWES site.
The unsteady boundary forcing and the changes of surface forcing (e.g., buoyancy) used in the simulations of the convective boundary layer result in the varying magnitude of budget terms over time. Figure 7 presents the TKE and various budget terms calculated using 30-min averages taken every $2 \mathrm{~h}$ (e.g., 1000-1030 PST, 1200-1230 PST) for the time period 1000-1430 PST in the fifth model layer ( $\approx 72 \mathrm{~m}$ above surface) for the CBWES site. The magnitude of the budget terms decreases with time from 1300 PST (not shown in Fig. 7), which is expected because of the decrease of the surface flux and associated decrease in the TKE production terms through the afternoon. The overall decrease in magnitude of all of the TKE budget terms (except dissipation) over time results in a gradual decrease of the TKE from 1000 to 1430 PST (first row of Fig. 7). An interesting feature is a common structure associated with the top of the ridge that is oriented diagonally from northwest to southeast and is clearly seen for all budget terms regardless of the time of day. The ridge is perpendicular to the mean wind direction, and a large amount of vertical wind shear is generated along the ridge when the southwesterly mean wind flows down the leeward side. In the later morning, as noticed in our previous work (Rai et al. 2017), the wind speed is smaller near the ridge top and larger in the lowelevation area (NE quadrant of the domain). Because of this difference in wind speed in space, a large negative advection of TKE along the ridge (large blue area in the first few panels in advection term) occurs. As time advances, the wind speed over the entire domain decreases, as do the area and magnitude of advection.

The pattern of the turbulent transport term reveals the effect of varying terrain elevation. However, the size and position of negative and positive areas of the advection term do not match with those of the transport term. For example, the advection has large positive values in the southwestern part of the domain because of large wind speed and wind shear, but the transport term shows large negative values in same area. This shows that advection is adding turbulence along the horizontal direction due to the large amount of wind-shear; conversely, the turbulence generated along the ridge is being transported elsewhere, most probably in the vertical direction, by the transport term as revealed by the small values of transport near the ridge. In this way, the interplay of the terrain and meteorological forcing (e.g., magnitude and direction of wind) may play an important role in the generation and movement of turbulence.

\section{d. Averaged TKE budget}

Horizontal spatial averaging of the entire budget profiles from WRF-LES domains within the area of each 


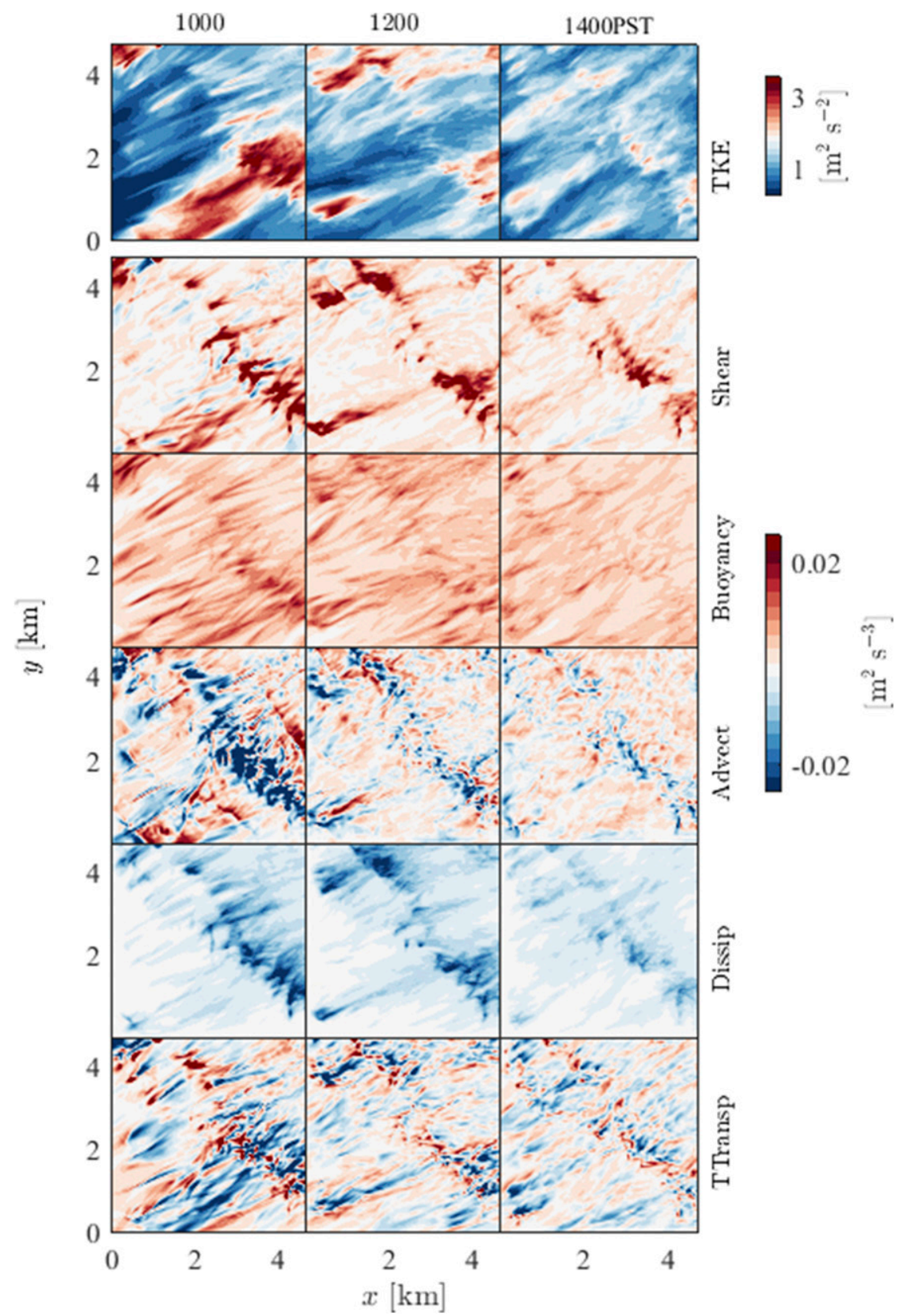

FIG. 7. Contours of TKE and various budget terms (30-min averaged data) in the horizontal plane of the fifth model layer ( $\approx 72 \mathrm{~m}$ above the surface) at every $2 \mathrm{~h}$ for the period $1000-1430$ PST for CBWES.

mesoscale grid cell may eliminate the variability seen in section 4c (see Fig. 8). Figure 8a (left panels) shows the spatially averaged profiles of each budget term in the four quadrants for the CBWES site. The area of each quadrant in the CBWES site is approximately $2.5 \mathrm{~km} \times 2.5 \mathrm{~km}$ $(82 \times 82$ grid points $)$ and consists of three types of terrain elevations-low (NE), high (SW), and mixed (NW, $\mathrm{SE})$. The results show that the magnitudes of spatially averaged budget terms (except buoyancy production) vary with the quadrant. For example, the NW and SE quadrants (mixed elevation) exhibit comparable profiles for total shear production, advection, and dissipation. However, in the other two quadrants, the averaged profile for these terms is found to be different because of the significant variability of turbulence (the dissipation is small in the NE and large in the SW). For each quadrant, 
a) CBWES site
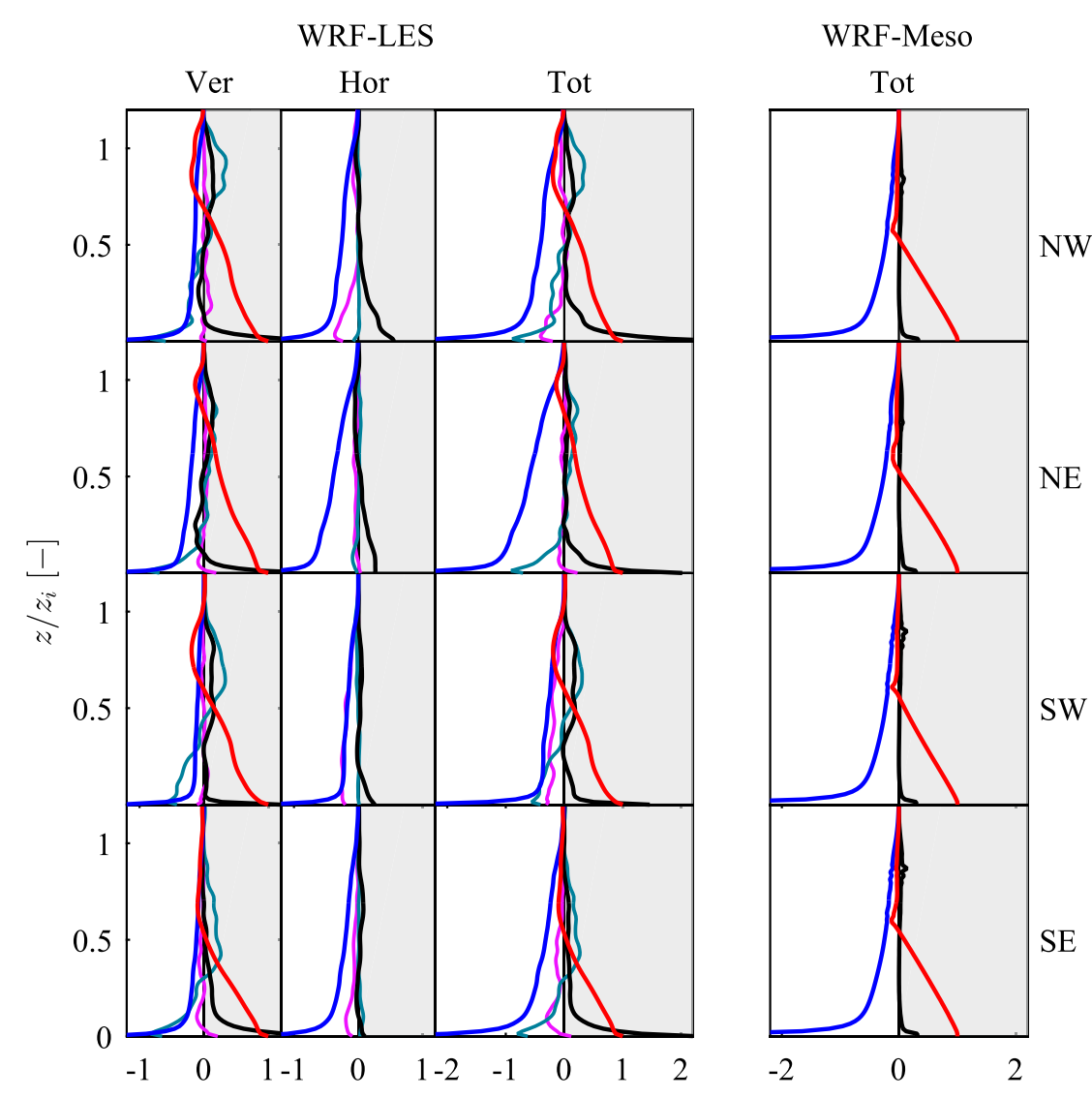

b) SWiFT site
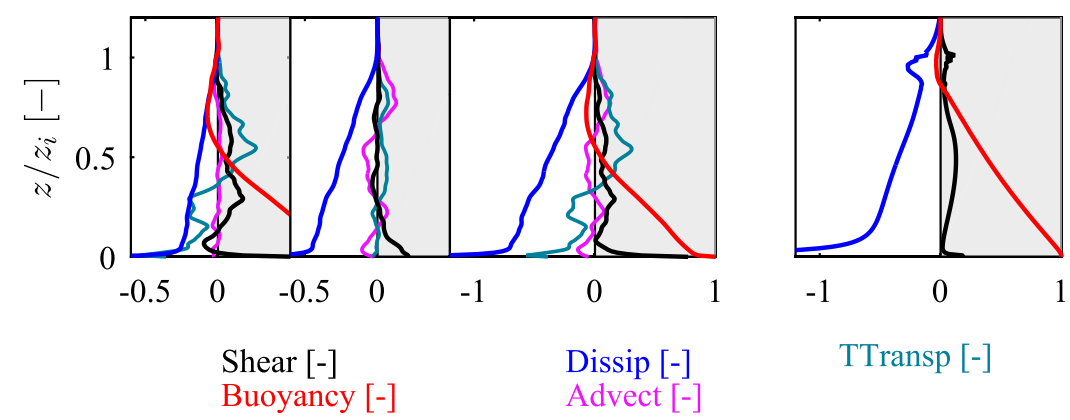

FIG. 8. TKE budget profiles at (a) the CBWES site (1300-1330 PST) in the NW, NE, SW, and SE quadrants and (b) the SWiFT site (1200-1230 CST) in the SW quadrant, obtained from averaging profiles of (left) LES grid cells of each quadrant and (right) output from mesoscale grid cells (domain D03). The labels Ver, Hor, and Tot represent the values obtained using vertical and horizontal derivatives and their total derivative. The gray and white areas are used to demarcate the positive and negative values of each budget term.

the nature of the total budget profiles, such as the negative buoyancy at the top of the boundary layer due to the entrainment and the vertical transport of turbulence, is found to be similar to that mentioned in a wide range of previous work (e.g., Moeng 1984; Moeng and Sullivan 1994). Considering the terms independently, the vertical
(Ver) and horizontal [Hor (sum along $x$ and $y$ directions)] derivative components associated with each of the budget terms reveal that both the horizontal and vertical derivatives contribute comparably to the total TKE tendency. Similarly, the averaged budget profiles show that shear is dominant over buoyancy in the regions very close 
to the surface. To compare with the results from the averaged TKE profile, the values of three budget terms (shear production, buoyancy production, and dissipation) for the CBWES site from four grid cells of mesoscale domain D03 are shown in Fig. 8a (right panels). Note that each grid cell of the mesoscale domain is approximately equal to the size of the one quadrant. The results show that budget terms from the grid cells of the mesoscale domain are almost identical to each other. Furthermore, the value of the shear production term near the surface from the mesoscale simulation is much smaller than the averaged TKE budget profile.

The budget profiles shown in Fig. 8a for the CBWES site are derived from the results of two different horizontal grid spacings [i.e., $30 \mathrm{~m}$ (WRF-LES) and $1.35 \mathrm{~km}$ (WRF-Meso)]. Furthermore, these two configurations use different resolution topography-30 s (i.e., for $1.35-\mathrm{km}$ grid spacing) and $1 \mathrm{~s}$ (i.e., for 30-m grid spacing)which could be partially responsible for the different amount of variability in the two domains. In addition to the topography, the variability could also be attributed to the better representation of processes within the boundary layer. Wagner et al. (2014) studied the influence of model grid spacing on the boundary layer structures in an idealized valley using resolved, smoothed, and underresolved topographies. They observed different structures in the boundary layer when using these varying treatments of the topography. In an additional LES run, using smoothed topography but keeping similar model grid spacing, they showed that differences in the boundary layer structures are mainly due to the unresolved topography and not the unresolved turbulence process. These findings indicate that the variability associated with both complex terrain and boundary layer physics may not be represented accurately by a mesoscale model using a turbulence parameterization designed for use over flat terrain.

Unlike in the CBWES site, the magnitudes of averaged profiles of budget terms from all four quadrants of the SWiFT site (e.g., Fig. $8 b$ for the SW quadrant) exhibit similar values to budget terms for both horizontal (Hor) and vertical (Ver) derivatives, as well as their sum. Figure $8 \mathrm{~b}$ (left panels) shows the profiles of budget terms for the SWiFT site derived from the WRF-LES data for domain D06. The area of each quadrant of the SWiFT site is a square of approximately $3.5 \mathrm{~km} \times 3.5 \mathrm{~km}(70 \times 70$ grid points). The vertical derivative of the shear term in the mid-PBL is found to be larger in magnitude than the horizontal derivative, which may be due to the large value of the buoyancy term. Similarly, a positive value of the advection term (near the top of the PBL) is seen in most of the quadrants for horizontal derivatives of the budget terms. The dominance of the buoyancy production term over shear production term throughout the PBL indicates that the turbulence in this case is predominantly generated by the buoyancy. The intensity of the shear and buoyancy production terms decreases with height within the PBL depth. As observed in the CBWES site, the large production (shear and buoyancy) of turbulence near the surface is transported to the upper half of the PBL depth, similar to that described in the literature (e.g., Moeng and Sullivan 1994). Figure 8b (right panel) shows the budget profiles from WRF-Meso data for the SW quadrant. One difference in the WRF-Meso profiles from the WRF-LES is the appearance of the bump in the dissipation term near the top of the boundary layer. The budget profile for the shear term shows that this bump in the dissipation term is partially due to larger wind shear at that location. Because the buoyancy term shows a negative value at this location, the remaining contribution to the bump in dissipation is associated with the transport and advection terms. Similar budget profiles from the remaining three grid cells of WRF domain D03 were obtained (not shown here). This suggests that there is less spatial variability in the magnitude of budget terms among the quadrants, and the mesoscale model is more or less able to reproduce the averaged budget profile in SWiFT site.

To evaluate the variability of the budget terms within a typical mesoscale grid cell, the entire budget profiles from each quadrant are used to calculate the standard deviations using time series at each height. Figures 9a and 9b show the standard deviations for the CBWES and SWiFT sites that were computed for each budget term in each of the four quadrants of the domain. The standard deviation was calculated using the horizontal and vertical derivative components (i.e., Hor and Ver) of the budget terms, as well as their sum. Each budget term was normalized using $w_{*}$, whereas the height was normalized using $z_{i}$. Our results show that the standard deviation due to the horizontal derivative of the advection term is found to be the largest, followed by the vertical derivative of the turbulent transport term for both sites. For both the horizontal and vertical derivatives, as well as their sum, the magnitude of standard deviation in the CBWES site is found to be larger than that computed for the SWiFT site. Comparing the standard deviation among the quadrants in the CBWES site, the NE quadrant shows the largest values. On the other hand, the SWiFT site has less variability of the standard deviation between the quadrants, most likely because of the flat terrain within that domain; this result confirms that there exists a great deal of variability within the area of a typical mesoscale grid box.

The integrated area associated with each budget profile (spatially averaged over the individual quadrants in Fig. 8) provides a quantity that can be used to evaluate the relative contribution of each budget term to the TKE 
a) CBWES (1300-1330 PST)

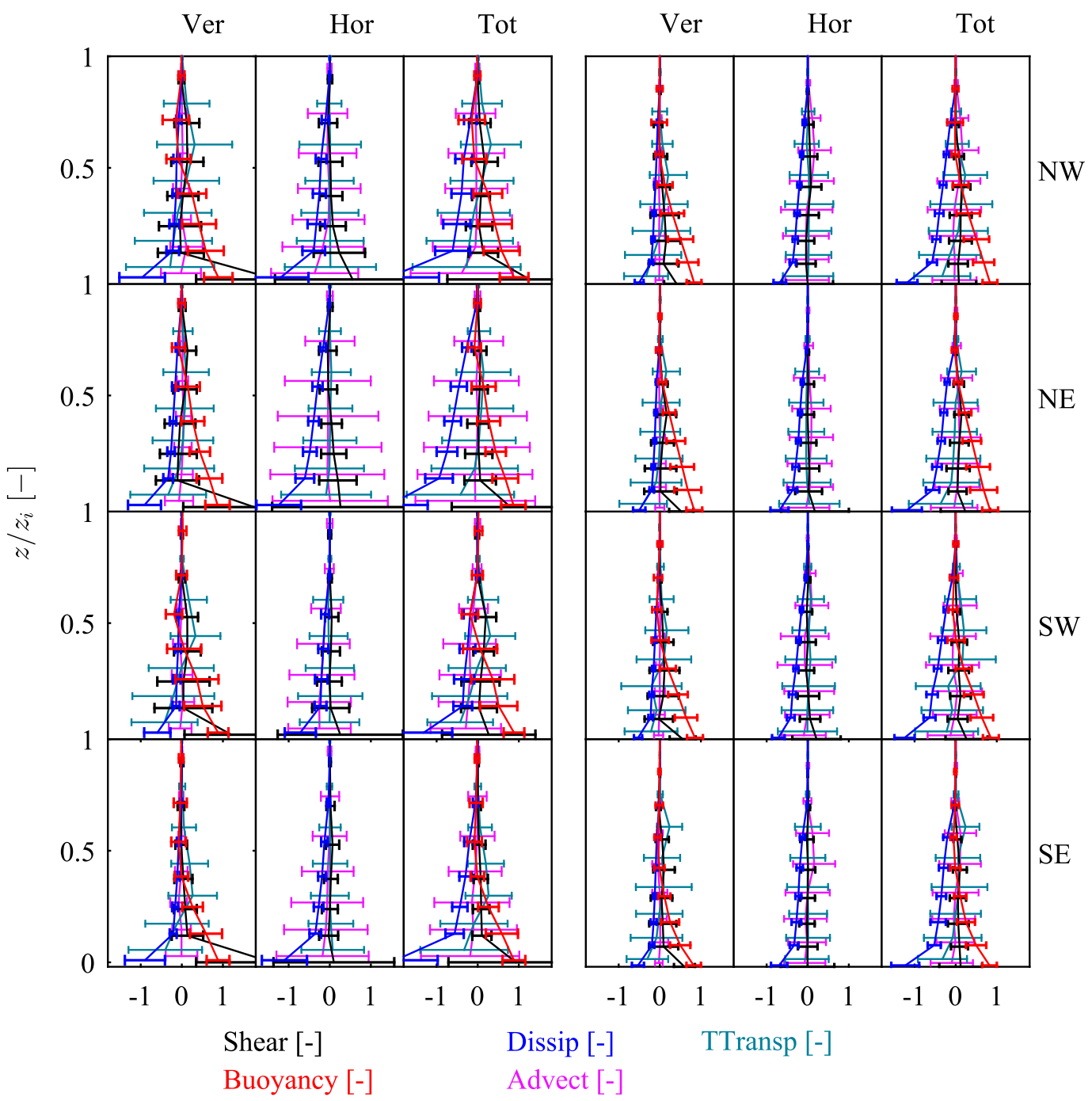

b) SWiFT (1200-1230 CST)

Ver Hor $\quad$ Tot

FIG. 9. Mean vertical profiles and error bars of budget terms (different colors) derived from vertical profiles in each quadrant for (a) CBWES and (b) SWiFT. The labels Ver, Hor, and Tot represent the terms obtained using vertical and horizontal derivatives and their total derivative.

tendency. For the profile of the sum, as well as for the horizontal and vertical derivatives of the TKE budget terms, Figs. 10a and 10b show the integrated area resulted from the profile (up to the PBL depth) over positive (gray) and negative (white) regions (refer to Fig. 8) for the CBWES and SWiFT sites. The integrated positive and negative areas in Fig. 10 show whether a particular budget term is adding or subtracting from the total amount of turbulence. The units of the integrated area presented in Fig. 10 (i.e., $\mathrm{m}^{3} \mathrm{~s}^{-3}$ ) were derived from the units associated with the budget term $\left(\mathrm{m}^{2} \mathrm{~s}^{-3}\right)$ and height $(\mathrm{m})$. At both sites, the buoyancy production is the dominant source of TKE, and it is nearly balanced by the dissipation over the depth of the PBL. Near the surface (particularly at the CBWES site), the magnitude of the shear production term is significant compared to the buoyancy production term. For example, the total shear production terms, by magnitude, for the NW, NE, SW, and SE quadrants at the CBWES site are approximately $85 \%, 40 \%, 65 \%$, and $60 \%$ of the total buoyancy production terms, respectively. On the other hand, the total shear production terms at the SWiFT site for the quadrants are found to be approximately $30 \%, 25 \%$, $30 \%$, and $40 \%$ of the total buoyancy production terms. Furthermore, the magnitude of shear production is nearly equal for the horizontal and vertical derivatives in each quadrant. The role of advection could also be different at the two sites: the decreasing mean wind, with time, at the 
a) CBWES (1300-1330 PST)
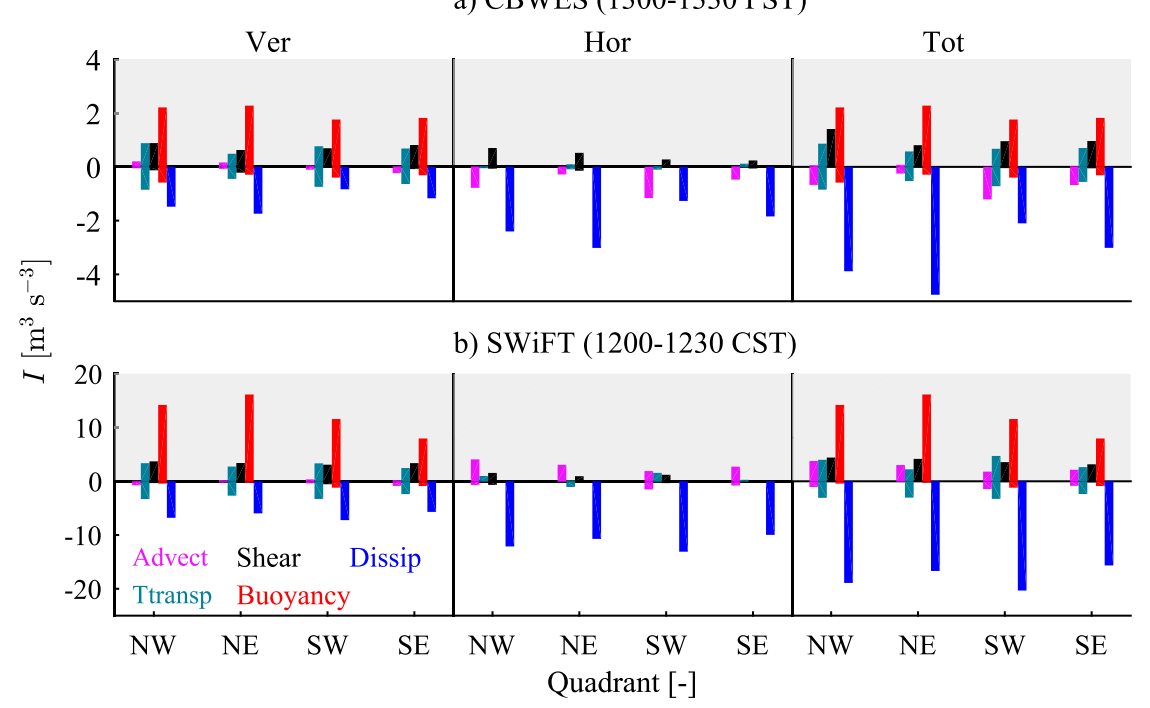

FIG. 10. Integrated value of the budget terms profile for the (a) CBWES and (b) SWiFT sites. The negative and positive values are obtained from integrating the profile under the negative and positive areas.

CBWES site could produce negative horizontal advection (i.e., reducing turbulence), whereas the increasing mean wind at the SWiFT site could result in positive advection (i.e., adding turbulence). At both sites, the advection in the horizontal derivative term is dominant over that found for the vertical derivative and is significant to the magnitude of the shear term. The turbulence transport is negligible in the horizontal direction, as its magnitude (positive and negative values) is distributed approximately equally along the vertical direction, thereby producing small net transport term values. This indicates pure vertical transport (with no horizontal contribution) of turbulence from the surface to the upper boundary layer, as discussed extensively in the literature (e.g., Moeng 1984; Moeng and Sullivan 1994; Weigel et al. 2007). The dissipation due to the horizontal derivative component is found to be larger than the dissipation due to the vertical derivative component.

The variability derived from WRF-LES data in the mesoscale footprint showed less variability among four mesoscale grids (i.e., quadrants) for the flat terrain site (i.e., SWiFT) compared to the complex terrain site (i.e., CBWES). This finding (from WRF-LES data) can be evaluated with the results from the WRF-Meso simulation, which uses the MYNN PBL parameterization for modeling turbulence in the vertical direction, as described in section 2. The TKE value from each grid cell (i.e., subgrid TKE) obtained from the WRF-Meso model can be compared with that derived from WRF-LES model data over the same geographic area and from the same time period. Figures 11a and $11 \mathrm{~b}$ show the vertical profiles of TKE obtained from WRF-Meso domain D03 and WRF-LES domain D06 for the CBWES and SWiFT sites, respectively, for the four quadrants. As in the previous figures, both the TKE and vertical heights are normalized using the convective velocity $\left(w_{*}\right)$ and boundary layer depth $\left(z_{i}\right)$. The results show that the parameterized TKE profiles from four grid cells of the WRF-Meso model differ from those of the quadrants of the WRF-LES model domain-overpredicting and underpredicting the TKE in the CBWES and SWiFT sites, respectively. In both sites, the shape and magnitude of the vertical TKE profiles from the WRFMeso model show good agreement with each other throughout the depth of the PBL. Similarly, the TKE profiles of the quadrants from WRF-LES data for the SWiFT site are similar to each other. However, the quadrants' shape and profiles among the TKE profiles derived from WRF-LES data for the CBWES site are found to be completely different. This behavior of TKE profiles between the two sites (CBWES and SWiFT) with different surface conditions confirms that the WRF-Meso model does not capture all of the relevant details of the turbulence associated with the variability observed in the complex terrain at the CBWES site. Inclusion of horizontal variability in the WRF-Meso model through parameterization of three-dimensional PBL could help address this issue.

\section{Summary and conclusions}

We have studied the spatial and temporal variability of the TKE budget over flat (SWiFT site) and complex terrain (CBWES site). The simulations utilized six nested domains (three each for mesoscale and LES) and 


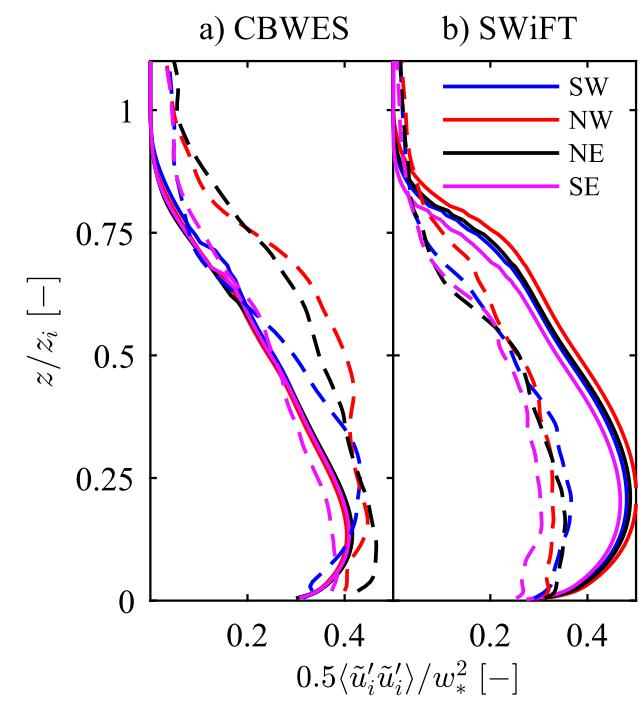

FIG. 11. Comparison of TKE derived from LES (resolved and modeled) variances (dashed lines) and mesoscale TKE, that is, subgrid output predicted by the WRF Model (solid lines) for (a) CBWES and (b) SWiFT in the four quadrants.

used the WRF Model framework to simulate convective boundary layers. The variability was assessed by analyzing the vertical profiles of the TKE budget terms in the two- and three-dimensional spaces.

The nondimensionalized profiles of the TKE budget computed at five locations for the CBWES site displayed more variability in magnitude at each vertical layer, compared to the budget profile for the SWiFT site. Similarly, the budget terms computed for vertical planes along and across the mean wind revealed a great deal of spatial variability at the CBWES site when compared with the SWiFT site. The CBWES site showed a large value of the shear production term near the surface in the vertical cross section crossing the main ridge. Similarly, the large dominant structures were seen in all budget terms near the main ridge in the horizontal cross section. In contrast, the budget terms for the SWiFT site did not reveal such spatiotemporal variability in the two-dimensional planes of the budget terms. This suggests that the terrain plays an important role for the generation and transport of turbulence in space.

The vertical profiles of the budget terms (derived from LES output) were used to calculate the mean and standard deviation at each vertical layer within each quadrant of the LES model domain $(\approx 1$ mesoscale grid cell). The mean profile of the budget terms for the CBWES site showed the varying magnitude among the quadrants, whereas the budget terms for the SWiFT site were similar to each other across the quadrants. The profile of the TKE budget terms calculated from each quadrant was compared against the TKE budget profiles from four of the host mesoscale model grid cells. The result showed that the profiles of the budget terms from each mesoscale grid cell for the CBWES site were nearly identical; however, their magnitudes were different than the mean profile of the budget terms derived for each quadrant from LES. On the other hand, the profiles of the budget terms for the SWiFT site from the mesoscale grid cells were nearly identical to each other and were similar to the mean budget profile derived for each quadrant from LES. The standard deviation of the budget terms within each quadrant showed a great deal of spatial variability regardless of the location. However, the magnitude varied even more, both among and within the quadrants, for the CBWES case. Overall, these analyses describe the effects of complex terrain on the spatial variability of the TKE budget, which is lacking many parameterizations applied in mesoscale models.

The dependence of the TKE budget terms on the horizontal and vertical derivatives was examined using their relative contribution to the total amount of TKE. In both complex and flat terrain cases, the LES output shows that the budget terms associated with the horizontal derivatives, such as the shear, are comparable in magnitude to the terms associated with the vertical derivatives. Similarly, the horizontal advection term makes a considerable contribution to the TKE budget. In mesoscale models, the horizontal derivatives are typically neglected, assuming that homogeneity exists in the horizontal direction, but these results show that is clearly not the case. The spatial variability seen at the two sites highlights that simulations completed using standard turbulence parameterizations applied in mesoscale models in complex terrain may not accurately simulate boundary layers and indicates the need for treatments that account for horizontal inhomogeneity, such as by three-dimensional PBL parameterization. The mesoscale PBL schemes with horizontal terms may represent the missing variability seen in the complex terrain case.

Acknowledgments. This research was supported by the Office of Energy Efficiency and Renewable Energy of the U.S. Department of Energy as part of the Wind Energy Technologies Office (Grant 28474/67023). The Pacific Northwest National Laboratory is operated for the DOE by Battelle Memorial Institute under Contract DE-AC05-76RLO1830.

\section{REFERENCES}

Berg, L. K., M. Pekour, and D. A. Nelson, 2012: Description of the Columbia Basin Wind Energy Study (CBWES). Pacific Northwest National Laboratory Tech. Rep. PNNL-22036, 24 pp., https://www.pnnl.gov/main/publications/external/ technical_reports/PNNL-22036.pdf.

Catalano, F., and C.-H. Moeng, 2010: Large-eddy simulation of the daytime boundary layer in an idealized valley using the Weather Research and Forecasting numerical model. Bound.-Layer Meteor., 137, 49-75, doi:10.1007/s10546-010-9518-8. 
Chen, F., and J. Dudhia, 2001: Coupling an advanced land surface-hydrology model with the Penn State-NCAR MM5 modeling system. Part I: Model implementation and sensitivity. Mon. Wea. Rev., 129, 569-585, doi:10.1175/ 1520-0493(2001)129<0569:CAALSH>2.0.CO;2.

Dwyer, M. J., E. G. Patton, and R. H. Shaw, 1997: Turbulent kinetic energy budgets from a large-eddy simulation of airflow above and within a forest canopy. Bound.-Layer Meteor., 84, 23-43, doi:10.1023/A:1000301303543.

Finnigan, J., 2000: Turbulence in plant canopies. Annu. Rev. Fluid Mech., 32, 519-571, doi:10.1146/annurev.fluid.32.1.519.

Iacono, M. J., J. S. Delamere, E. J. Mlawer, M. W. Shephard, S. A. Clough, and W. D. Collins, 2008: Radiative forcing by longlived greenhouse gases: Calculations with the AER radiative transfer models. J. Geophys. Res., 113, D13103, doi:10.1029/ 2008JD009944.

Kelley, C. L., and B. L. Ennis, 2016: SWiFT site atmospheric characterization. Sandia National Laboratories Tech. Rep. SAND2016-0216, 82 pp., http://prod.sandia.gov/techlib/ access-control.cgi/2016/160216.pdf.

Lenschow, D. H., 1974: Model of the height variation of the turbulence kinetic energy budget in the unstable planetary boundary layer. J. Atmos. Sci., 31, 465-474, doi:10.1175/ 1520-0469(1974)031<0465:MOTHVO > 2.0.CO;2.

Lilly, D. K., 1967: The representation of small-scale turbulence in numerical simulation experiments. Proc. IBM Scientific Computing Symp. on Environmental Sciences, Yorktown Heights, NY, International Business Machines Corporation, 195-210.

McBean, G., and J. Elliott, 1975: The vertical transports of kinetic energy by turbulence and pressure in the boundary layer. J. Atmos. Sci., 32, 753-766, doi:10.1175/1520-0469(1975)032<0753: TVTOKE $>2.0 . \mathrm{CO} ; 2$.

Mesinger, F., and Coauthors, 2006: North American Regional Reanalysis. Bull. Amer. Meteor. Soc., 87, 343-360, doi:10.1175/ BAMS-87-3-343.

Mirocha, J., J. Lundquist, and B. Kosović, 2010: Implementation of a nonlinear subfilter turbulence stress model for large-eddy simulation in the Advanced Research WRF model. Mon. Wea. Rev., 138, 4212-4228, doi:10.1175/2010MWR3286.1.

Moeng, C.-H., 1984: A large-eddy-simulation model for the study of planetary boundary-layer turbulence. J. Atmos. Sci., 41, 2052-2062, doi:10.1175/1520-0469(1984)041<2052:ALESMF>2.0.CO;2.

— , and P. P. Sullivan, 1994: A comparison of shear- and buoyancy-driven planetary boundary layer flows. J. Atmos. Sci., 51, 999-1022, doi:10.1175/1520-0469(1994)051<0999: $\mathrm{ACOSAB}>2.0 . \mathrm{CO} ; 2$.

Morrison, H., G. Thompson, and V. Tatarskii, 2009: Impact of cloud microphysics on the development of trailing stratiform precipitation in a simulated squall line: Comparison of one- and two-moment schemes. Mon. Wea. Rev., 137, 991-1007, doi:10.1175/2008MWR2556.1.

Nakanishi, M., and H. Niino, 2006: An improved Mellor-Yamada level-3 model: Its numerical stability and application to a regional prediction of advection fog. Bound.-Layer Meteor. 119, 397-407, doi:10.1007/s10546-005-9030-8.

Rai, R. K., L. K. Berg, B. Kosović, J. D. Mirocha, M. S. Pekour, and W. J. Shaw, 2017: Comparison of measured and numerically simulated turbulence statistics in a convective boundary layer over complex terrain. Bound.-Layer Meteor., 163, 69-89, doi:10.1007/s10546-016-0217-y.

Skamarock, W. C., 2004: Evaluating mesoscale NWP models using kinetic energy spectra. Mon. Wea. Rev., 132, 3019-3032, doi:10.1175/MWR2830.1.

— , and Coauthors, 2008: A description of the Advanced Research WRF version 3. NCAR Tech. Note NCAR/TN-475+STR, 113 pp., doi:10.5065/D68S4MVH.

Skyllingstad, E. D., 2003: Large-eddy simulation of katabatic flows. Bound.Layer Meteor., 106, 217-243, doi:10.1023/A:1021142828676.

Smagorinsky, J., 1963: General circulation experiments with the primitive equations: I. The basic experiment. Mon. Wea. Rev., 91, 99-164, doi:10.1175/1520-0493(1963)091<0099: GCEWTP $>2.3 . C O ; 2$.

Stull, R. B., 1988: An Introduction to Boundary Layer Meteorology. Atmospheric and Oceanographic Sciences Library, Vol. 13, Springer, $670 \mathrm{pp}$.

Wagner, J. S., A. Gohm, and M. W. Rotach, 2014: The impact of horizontal model grid resolution on the boundary layer structure over an idealized valley. Mon. Wea. Rev., 142, 3446-3465, doi:10.1175/MWR-D-14-00002.1.

Weigel, A. P., F. K. Chow, and M. W. Rotach, 2007: On the nature of turbulent kinetic energy in a steep and narrow Alpine valley. Bound.-Layer Meteor., 123, 177-199, doi:10.1007/s10546-006-9142-9.

Wyngaard, J. C., 2004: Toward numerical modeling in the "terra incognita." J. Atmos. Sci., 61, 1816-1826, https://doi.org/10.1175/ 1520-0469(2004)061<1816:TNMITT>2.0.CO;2.

— and temperature variance in the atmospheric surface layer. J. Atmos. Sci. 28, 190-201, doi:10.1175/1520-0469(1971)028<0190: TBOTKE $>2.0 . \mathrm{CO} ; 2$.

Yang, B., A. P. Morse, R. H. Shaw, and K. T. Paw U, 2006: Largeeddy simulation of turbulent flow across a forest edge. Part II: Momentum and turbulent kinetic energy budgets. Bound.-Layer Meteor., 121, 433-457, doi:10.1007/s10546-006-9083-3.

Yue, W., C. Meneveau, M. B. Parlange, W. Zhu, H. S. Kang, and J. Katz, 2008: Turbulent kinetic energy budgets in a model canopy: Comparisons between LES and wind-tunnel experiments. Environ. Fluid Mech., 8, 73-95, doi:10.1007/ s10652-007-9049-0. 